Cahiers de la recherche sur les droits

Cahiers

Fur les Droits fondamentaux

$8 \mid 2010$

La liberté d'expression

\title{
Le droit français et la protection des Roms
}

\section{Catherine-Amélie Chassin}

\section{OpenEdition}

Journals

Édition électronique

URL : https://journals.openedition.org/crdf/6182

DOI : $10.4000 /$ crdf.6182

ISSN : 2264-1246

Éditeur

Presses universitaires de Caen

\section{Édition imprimée}

Date de publication : 20 décembre 2010

Pagination : 135-146

ISBN : 978-2-84133-367-7

ISSN : 1634-8842

Référence électronique

Catherine-Amélie Chassin, « Le droit français et la protection des Roms », Cahiers de la recherche sur les droits fondamentaux [En ligne], 8 | 2010, mis en ligne le 08 octobre 2020, consulté le 15 novembre 2022. URL : http://journals.openedition.org/crdf/6182 ; DOI : https://doi.org/10.4000/crdf.6182 


\title{
Le droit français et la protection des Roms ${ }^{1}$
}

\author{
Catherine-Amélie CHASSIN
}

Maître de conférences (HDR) en droit public à I'Université de Caen Basse-Normandie

Centre de recherche sur les droits fondamentaux et les évolutions du droit (CRDFED)

I. Les Roms français

A. Le hiatus juridique : des Roms invisibles mais dotés d'un statut particulier

1. Les Roms, entité invisible au sein du peuple français

2. L'assimilation des Roms aux "gens du voyage"

B. Un accès limité aux droits

1. Les restrictions induites par le statut de gens du voyage

2. La question du droit au logement et la Charte sociale européenne

II. Les Roms étrangers

A. Un régime fonction de l'État d'origine

1. Les Roms, ressortissants communautaires

a. Le principe de libre circulation

b. Les Roms communautaires exerçant une activité professionnelle

2. Les Roms venant d'un État tiers

B. La difficile éligibilité des Roms au statut de réfugié

1. Les Roms et la notion de pays d'origine sûr

2. Quelle(s) persécution(s) pour les Roms?

La question de la protection des populations roms par le droit français est tout à la fois vaste et simple. Vaste, parce que les discriminations dont sont victimes les Roms sont aussi présentes en France qu'ailleurs; simple, parce que le droit français fait souvent l'impasse sur la spécificité des populations concernées, faute de dispositions juridiques particulières.
La situation des populations roms en France pourrait être résumée, dans une large mesure, par les propos tenus en octobre 2002 par le préfet Paul Girot de Langlade, alors représentant de l'État dans le département du Vaucluse, et publiés par le journal régional La Provence: "Je n'ai aucune tendresse particulière pour ces gens-là. Ils vivent à nos crochets, de la rapine, aussi, tout le

1. La présente contribution a été rédigée dans le cadre des Universités d'été organisées par la Faculté de droit de Belgrade (Serbie), en septembre 2009. L'auteur tient à remercier ici le Professeur Branko Rakic et Maja Lukic, enseignants à la Faculté de Belgrade, mais aussi le doyen Jean-François AkandjiKombé et Sébastien Botreau-Bonneterre. 
monde le sait [...]. Certains roulent dans des Mercedes que je ne pourrais même pas me payer. Moi aussi, ça m'agace $^{2}$.»

Cette virulente sortie avait été condamnée par le Gouvernement français par la voie de son ministre de l'Intérieur, Nicolas Sarkozy; reste que ces propos représentent, assez largement, l'état de l'opinion publique française vis-à-vis des populations roms. Un mélange d'incompréhension, de rejet et d'a priori, non fondés mais persistants.

Une preuve de cette forme pour le moins condamnable d'appréhension des Roms se trouve sans doute dans l'attitude de la Gendarmerie nationale à Corbeil-Essonnes, dans la banlieue parisienne, le 28 août 2009 . Les gendarmes étaient appelés à interpeller des ressortissants étrangers roms, en vue de l'exécution forcée des mesures d'éloignement du territoire qui avaient été adoptées contre certains d'entre eux. Ces personnes se trouvaient vivre dans un bidonville relativement peuplé. Afin de distinguer ceux qui avaient déjà été contrôlés de ceux qui ne l'étaient pas encore, les forces de l'ordre n'ont rien trouvé de mieux, pour identifier les premiers, que de leur apposer un coup de tampon administratif sur l'avant-bras. Mauvais souvenir pour une population qui, comme les juifs, connut trop souvent la déportation durant la seconde guerre mondiale; atteinte indéniable aux principes les plus élémentaires de la dignité de la personne humaine, les Roms étant ici rabaissés au rang de ces animaux que l'on tatoue pour les identifier plus facilement, en vue de les trier pour l'abattoir. Tel n'était pas le destin des Roms ciblés, reste que la méthode est pour le moins choquante.

Ce qui frappe, dans cette affaire, est certes le moyen utilisé par les gendarmes, et qui fut critiqué par le ministre de l'Intérieur et par celui de l'Immigration. Mais peutêtre aussi, et surtout, ce qui étonne est que les médias français, pourtant généralement prompts à réagir, n’ont que rarement et tardivement jugé utile de se faire le relais de ce qui s'était produit. L'affaire n'est apparue dans les médias que trois semaines plus tard, parce qu'une émission satyrique y avait fait allusion ; à défaut, nul doute que la presse française aurait continué d'ignorer ces pratiques, et que l'opinion publique, nonobstant son droit à l'information consacré par ailleurs par la Cour européenne des Droits de l'homme ${ }^{3}$, n'aurait jamais rien su des contrôles de Corbeil-Essonnes. Un tel comportement, tant celui de la gendarmerie de l'Essonne que celui des médias français, marque un désintérêt certain envers le sort des populations roms. Car on n'imagine guère une attitude similaire envers d'autres (étrangers ou nationaux) passer inaperçue, que ce soit d'ailleurs aux yeux de l'opinion publique française qu'à ceux de l'État d'origine des intéressés. Cette déplorable affaire marque ce que sont les Roms aujourd'hui : des populations ignorées et, partant, exposées à toutes sortes de discriminations et de raccourcis délétères. Ainsi un sénateur français avait-il pu, lors d'une séance de questions au Gouvernement en 2001, établir un lien explicite entre le niveau de délinquance et la présence de population roms ${ }^{4}$ - et ce à nouveau sans que personne ne s'en offusque particulièrement. De façon générale, et ainsi que l'Agence européenne des droits fondamentaux l'a relevé dans un rapport de novembre 2009, les Roms sont objet de stéréotypes qui les assimilent à une source de problèmes à venir ${ }^{5}$.

La présence rom en France aujourd'hui est à l'image de cette ignorance. Et cela va jusqu'à leur nombre : selon les estimations, fort variables, ils seraient entre quelques dizaines de milliers et 300 ooo sur le sol de la République. La difficulté de l'estimation tient, ici, à deux phénomènes centripètes : d'une part, les Roms qui ont la nationalité française ne sont, en raison du droit interne, pas identifiables en tant que Roms; d'autre part, ceux qui n'ont pas la nationalité française sont souvent en situation irrégulière et partant largement invisibles aux yeux des autorités. Ces deux facteurs rendent tout chiffrage pour le moins hasardeux.

Néanmoins, le droit français ne peut totalement ignorer leur situation. Il le fait à travers le prisme de la nationalité avec, d'un côté, le traitement des Roms français (I) et, de l'autre, le statut des Roms étrangers (II).

\section{Les Roms français}

Le premier cas de figure qui nous retiendra sera donc celui des Roms qui ont la nationalité française, que ce soit par attribution ou par acquisition. On pourrait penser que, en tant que citoyens français, ils ne sont pas l'objet de discriminations. Et, pourtant, une analyse de la réalité démontre que les Roms, fussent-ils français, restent des citoyens particuliers (B), et ce malgré l'absence de visibilité officielle (A).

2. Compte rendu de la conférence de presse tenue le 23 octobre 2002, publiée dans La Provence, édition du 24 octobre.

3. Voir notamment l'arrêt de la Cour EDH, 23 septembre 1994, Jersild c. Danemark, 15890/89, \$31.

4. Le sénateur Pierre Hérisson avait, lors des questions au Gouvernement, tenu les propos suivants: «la France, ses élus, ses forces de police, sa justice, ainsi que l'ensemble de nos concitoyens, sont aujourd'hui en situation d'insécurité notoire, dans la crainte et dans la peur, et pour certains, les plus exposés comme les forces de police, en danger de mort [...] Que comptez-vous faire, monsieur le ministre? Il y a extrême urgence, il s'agit d’un véritable péril social. » Voir le compte rendu de la séance du 10 mai 2001, «Questions d'actualité au Gouvernement», JORF.Q, 10 mai 2001, p. 49. Pour l'anecdote, M. Girot de Langlade, devenu préfet d'Indre-et-Loire, avait tenu des propos similaires en novembre 2006; l'affaire s'était, alors, soldée par une condamnation, par le Tribunal de grande instance de Paris le 13 septembre 2007, du chef d'incitation à la discrimination raciale.

5. Voir The situation of Roma EU citizens moving to and settling in other EU Member States, rapport édité par l'Agence européenne des droits fondamentaux (FRA, European Union Agency for Fundamental Rights, Vienne), 9 novembre 2009, p. 8 : « Roma from other Member States are often stereotyped as causing problems and rarely positively welcomed. There is some evidence to suggest that their experience of settling in a new country has lead to immiseration rather than opportunity. This is, of course, a relative and subjective concept, but the research suggests that some Roma from other Member States may find themselves even more marginalised than they were in their country of origin.» 


\section{A. Le hiatus juridique : des Roms invisibles mais dotés d'un statut particulier}

Le droit français réussit ce qui pourrait, de prime abord, sembler infaisable : si l'entité rom n'est pas visible en tant que telle (1), les Roms français se voient pourtant, dans leur très grande majorité, imposer un statut exorbi$\operatorname{tant}(2)$.

\section{Les Roms, entité invisible au sein du peuple français}

Un Rom qui possède la nationalité française n'est pas visible, eu égard au droit interne. Cette curiosité nationale tient à l'article $1^{\mathrm{er}}$ de la Constitution du 4 octobre 1958, ainsi formulé : «La France est une république indivisible, laïque, démocratique et sociale. Elle assure l'égalité devant la loi de tous les citoyens sans distinction d'origine, de race ou de religion. Elle respecte toutes les croyances. Son organisation est décentralisée. »

Des premiers mots se déduit la notion, héritée de la Révolution française, d'une république "une et indivisible $»^{6}$. Si cela induit l'existence d'un État unitaire, ce n'est pas sur ce point que le texte mérite que l'on s'attarde, mais sur son implication en ce qui concerne la population française. La notion de minorité n'est pas admise par le droit interne, qui ne connaît qu'un seul et même peuple français. Saisi d'un recours contre une loi qui mentionnait l'existence, au sein du peuple français, du peuple corse, le Conseil constitutionnel a, par une décision du 9 mai 1991, rejeté avec force cette notion, considérant que la Constitution «ne connaît que le peuple français, composé de tous les citoyens français, sans distinction d'origine, de race ou de religion $"{ }^{7}$.

Dès lors, il devient impossible, au sein des Français, de distinguer ceux qui seraient Roms de ceux qui ne le seraient pas, dans la mesure où, au nom du modèle universaliste, tous sont français. Leur identification juridique est donc impossible, toute mention à leur origine étant prohibée. C'est d'ailleurs ce dogme d'une unicité du peuple français qui explique les réticences de la France à prendre toutes sortes d'engagements internationaux relatifs aux minorités: ainsi la France a-t-elle refusé de ratifier la Convention pour la protection des minorités nationales de $1995^{\circ}$; de même n'a-t-elle pas ratifié la Charte sur la protection des langues minoritaires de $1992^{9}$.

Et pourtant, au printemps 2009, le ministre en charge de la Diversité, Yazid Sabeg, avait suggéré d'introduire la possibilité d'établir, en France, des statistiques ethniques qui auraient notamment eu le mérite de mettre en exergue les discriminations dont certains Français peuvent être victimes, que ce soit au sujet de leur couleur, de leur religion ou de leur origine. L'idée avait, en mars et avril 2009, créé un débat assez virulent, mais qui a tourné court: à l'occasion d'un remaniement gouvernemental, en juin, M. Sabeg a dû quitter ses fonctions; l'idée des statistiques ethniques a disparu avec lui. Et l'on pourrait citer, dans la même perspective, le débat qui exacerbe certaines passions en cet hiver 2009-2010 autour de l'idée d'«identité nationale»: la notion de Français est ici appréhendée comme un bloc monolithique, dans lequel devraient se fondre tous les éventuels particularismes.

Ces exemples confirment l'invisibilité des Roms en tant que minorités: ne pouvant se présenter comme «Français roms", ils ne peuvent être que français, leur spécificité étant inéluctablement gommée par leur nationalité. L'assimilation induit ici une sorte d'épuration culturelle.

\section{L'assimilation des Roms aux " gens du voyage "}

Les Roms ne sont pourtant pas des Français comme les autres. Ainsi que cela est régulièrement relevé, tant d'ailleurs par les ONG que par le commissaire aux Droits de l'homme du Conseil de l'Europe, les Roms français conservent souvent un habitat mobile - bien qu'ils aient, dans leur majorité, fait le choix d'une installation à long terme. De ce fait, la plupart de ces Roms français sont assimilés aux "gens du voyage », ce qui leur confère un statut particulier ${ }^{10}$.

Organisé aujourd'hui par une loi de juillet $2000^{11}$, le statut des gens du voyage impose aux intéressés des obligations propres, ce qui aboutit à en faire des citoyens à part. Ils doivent notamment être en possession d'un carnet ou d'un livret de circulation - carnet qui doit être régulièrement visé par les autorités de police ou de gendarmerie $^{12}$. Ils sont de surcroît soumis à un quota de $3 \%$

6. La notion est utilisée dès la première Constitution écrite qu'a connue la France, la Constitution monarchique des 3-14 septembre 1791, dont l'article $1^{\text {er }}$ du titre II précisait : «Le Royaume est un et indivisible. »

7. Conseil constitutionnel, décision $n^{\circ}$ 91-290 DC du 9 mai 1991, Collectivité territoriale de Corse, $13^{\mathrm{e}}$ considérant. Voir, entre autres, sur ce point F. Luchaire et L. Favoreu, «À propos du statut de la Corse », Revue française de droit constitutionnel, n ${ }^{\circ}$, 1991, p. 484-491; C. Grewe, «Le nouveau statut de la Corse devant le Conseil constitutionnel », Revue universelle des Droits de l'homme, vol. 3, n 5-6, 1991, p. 381-389; B. Genevois, «Le contrôle de la constitutionnalité du statut de la collectivité territoriale de Corse », Revue française de droit administratif, n ${ }^{\circ} 3,1991$, p. 407-423.

8. La Convention-cadre pour la protection des minorités nationales (STCE nº 157$)$ a été adoptée à Strasbourg le $1^{\text {er }}$ février 1995 . La France ne l’a même pas signée.

9. La Charte européenne des langues minoritaires (STCE $n^{\circ}$ 148), datée du 5 novembre 1992, a été signée par la France en 1999, mais non pas ratifiée. Une révision de la Constitution française, en juillet 2008, a introduit dans le texte fondamental un article 75-1 relatif aux langues régionales; mais rien n'est préjugé sur le statut des langues minoritaires - tel le romani, propre aux Roms mais qui ne saurait pour autant être assimilé à une langue régionale.

10. Pour une étude globale, voir C. Le Berre, «Les gens du voyage: une catégorie ambiguë, source de discrimination indirecte», Revue du droit public, $\mathrm{n}^{\mathrm{o}} 124$, vol. 2, 2008, p. 891-920.

11. Loi $\mathrm{n}^{\circ}$ 2000-614 du 5 juillet 2000 sur l'accueil et l'habitat des gens du voyage, Journal officiel de la République française (JORF), 6 juillet 2000 , p. 10189. La constitutionnalité de la loi a été confirmée par le Conseil constitutionnel, déc. 2010-13 QPC du 9 juillet 2010, MM. Opra et Balta.

12. Cette obligation avait été renouvelée par une loi 69-3 du 3 janvier 1969, relative à l'exercice des activités ambulantes et au régime applicable aux personnes circulant en France sans domicile ni résidence fixe. Le livret de circulation est obligatoire pour les Français exerçant une activité ambulante 
de la population de la commune sur le territoire de laquelle ils s'installent, ce qui est tout à la fois paradoxal (s'agissant de Français, censés être tous égaux) et discriminatoire (envers ceux qui ont fait le choix de ce mode d'habitat, quelles qu'en soient les raisons profondes). En outre, ce régime dérogatoire ne dispense pas les intéressés des obligations fiscales, dans la mesure où, en dépit de leur mode d'habitat, ils restent soumis à une taxe annuelle d'habitation depuis la loi de finances pour 2005.

La difficulté est que ce qui caractérise ces gens du voyage n'est pas le nomadisme présupposé; ils tiennent ce régime juridique particulier du mode de leur habitat traditionnel, qui prend souvent la forme d'un habitat mobile, et ce alors même que, bien souvent, ils sont sédentarisés de longue date. C'est là une solution qui s’inscrit dans la lignée de la loi précitée de 2000 qui évoquait un mode d'habitat «traditionnel ${ }^{13}$, mais qui reste difficile à justifier pour des populations sédentaires.

L'assimilation des Roms français aux gens du voyage est récurrente et a été relevée explicitement par le commissaire aux Droits de l'homme du Conseil de l'Europe, dans son rapport de 2006 sur le respect des Droits de l'homme en France $^{14}$. Et de fait, on ne peut que constater que les propositions d'aménagement du statut rejettent toute spécificité des Roms, englobant sous un vocable administratif unique et un régime juridique commun une large hétérogénéité de situations ${ }^{15}$.

Dès lors, les Roms se trouvent, quoique Français, soumis à un régime juridique dérogatoire, qui les prive de certains droits pourtant essentiels et les maintient, de facto, dans un état d'infériorité.

\section{B. Un accès limité aux droits}

Le problème posé par l'assimilation des Roms aux gens du voyage est celui de l'accès aux droits de cette catégorie de Français. Au-delà des restrictions induites par le statut même de gens du voyage (1), apparaît la question particulière du droit au logement et de la conformité de la situation française avec ses engagements européens, notamment la Charte sociale européenne (2).

\section{Les restrictions induites par le statut de gens du voyage}

La difficulté principale opposée aux Roms est que, pris en tant que gens du voyage, ils n'ont pas de domicile foncier. Ils sont, dès lors, contraints de s'en voir reconnaître un par le biais de leur rattachement à une commune. Or, ce rattachement ne peut être effectif qu'à partir du moment où l'intéressé s'est déclaré durant trois années consécutives auprès de la même commune. Ainsi, durant les trois premières années de leur installation, ils ne peuvent être considérés comme habitants de ladite commune; et le moindre déménagement aboutira, inéluctablement, à la mise en place de la même période de vacance de trois ans. Or, le rattachement à une commune est le prélude indispensable pour l'exercice d'un certain nombre de droits. Il y a ici un véritable effet domino, la domiciliation étant l'élément clé pour pouvoir bénéficier d'un certain nombre de droits pourtant fondamentaux, à commencer par l'exercice effectif du droit de vote.

À ce premier point s'y ajoutent des difficultés concrètes moins voyantes mais tout aussi contraignantes. Ainsi de la scolarisation des enfants : si le droit à l'éducation est garanti par les textes ${ }^{16}$, sa mise en œuvre est rendue plus hasardeuse dès lors que les parents ne peuvent justifier d'un domicile reconnu sur le territoire d'une commune. L'intégration d'un enfant dans une école maternelle impose en effet, en France, une inscription préalable en mairie ${ }^{17}$; or, la commune exige, parmi les documents à fournir en vue de cette inscription, la production d'un justificatif récent de domicile. Ainsi, les Roms se heurtent à deux séries de difficultés concrètes : d'une part, le rattachement à une commune, qui permet effectivement d'obtenir de celle-ci la scolarisation de l'enfant; d'autre part, la production d'un justificatif concernant un domicile pour lequel il n'existe pas de rattachement administratif reconnu. Certes, ces difficultés ne sont pas insurmontables, mais elles sont systématiques et conduisent à une fragilisation certaine des populations concernées.

C'est là un aspect souvent méconnu par les autorités elles-mêmes, en dépit des efforts développés ${ }^{18}$. Ainsi la circulaire du 20 mai $1999^{19}$, si elle se penche sur la question

rémunératrice; les gens du voyage n'exerçant pas une telle activité sont, eux, soumis au carnet de circulation. Une loi récente du 4 août 2008 est venue alléger très partiellement le régime, en supprimant l'obligation de faire déclaration, auprès des autorités municipales, de son activité ambulante (loi $\mathrm{n}^{\mathrm{o}}$ 2008-776 dite de modernisation de l'économie, article 53, JORF, 5 août 2008, p. 12471).

13. Article $1^{\text {er }}$ de la loi : «les communes participent à l'accueil des personnes dites gens du voyage, et dont l'habitat traditionnel est constitué de résidences mobiles".

14. Rapport d'A. Gil Robles sur le respect effectif des Droits de l'homme en France, 15 février 2006, $\$ 330$.

15. Voir notamment la proposition de loi n 474 présentée au Sénat le 17 juin 2009, relative aux obligations de l'État en matière d'aires d'accueil des gens du voyage.

16. Art. L. 111-1 du Code de l'éducation (C. éduc.), alinéa 5 : « le droit à l'éducation est garanti à chacun afin de lui permettre de développer sa personnalité, d'élever son niveau de formation initiale et continue, de s'insérer dans la vie sociale et professionnelle, d'exercer sa citoyenneté ».

17. Art. L. 131-6 C. éduc., alinéa $1^{\mathrm{er}}$ : « chaque année, à la rentrée scolaire, le maire dresse la liste de tous les enfants résidant dans sa commune et qui sont soumis à l'obligation scolaire».

18. En particulier par la mise en place de structures propres. En 1975 avaient été créées des structures aidant à l'accueil des enfants de migrants; en 2002, les CASNAV (Centres académiques pour la scolarisation des nouveaux arrivants et des enfants du voyage) ont pris le relais. Ces structures permettent notamment l'insertion des enfants du voyage dans les classes ordinaires, en assurant la consolidation des acquis scolaires élémentaires.

19. Circulaire du 20 mai 1999 sur le contrôle de l'obligation scolaire, publiée au Bulletin officiel de l'enseignement, hors série $\mathrm{n}^{\circ} 3$, 20 mai 1999 , disponible sur www.education.gouv.fr/bo/1999/hs3/default.htm. 
des enfants du voyage (point III.3), rappelle aussi l'obligation, pour les parents, d'envoyer leur(s) enfant(s) fréquenter l'établissement scolaire de la commune sur le territoire de laquelle ils séjournent; elle rappelle également le fait que le maire ne peut refuser d'inscrire un enfant du voyage dans l'enseignement primaire. Mais rien n'est préjugé des difficultés matérielles que rencontrent les parents concernés, ni de la question de l'inscription dans un établissement scolaire secondaire. Et, surtout, le statut actuel assimile ces enfants aux enfants de personnes sans domicile fixe $\mathrm{e}^{20}$, sans considération du fait qu'ils ont, eux, un domicile.

Une évolution était donc attendue, mais la circulaire du 25 avril $2002^{21}$ reste en deçà des espérances et n'améliore que partiellement la situation. Il est en effet rappelé que l'enfant bénéficie, faute de justification du domicile sur le territoire de la commune, d'un accueil provisoire; mais ce n'est, précisément, qu'un accueil provisoire, « dans l'attente de la présentation, dans les plus courts délais, de ces documents qui permettront d'effectuer l'inscription de l'enfant à l'école ». Il ne s'agit pas, pour autant, d'une scolarisation pérenne des enfants concernés ${ }^{22}$.

L'assimilation des Roms français aux gens du voyage limite, ainsi que nous l'avons vu à travers le prisme de la scolarisation ${ }^{23}$, l'accès à un certain nombre de droits fondamentaux ${ }^{24}$. Mais elle entraîne également des difficultés en matière d'accès au droit au logement.

\section{La question du droit au logement et la Charte sociale européenne}

Le droit communautaire interdit toute discrimination dans l'accès au logement ; le principe en a été clairement posé par la directive du 29 juin $2000^{25}$. Mais c'est dans le domaine du droit européen que s'est posée la question de la discrimination des gens du voyage (Roms français, mais aussi d'ailleurs étrangers) en matière de droit au logement, par le biais d'une réclamation collective déposée par le Centre européen pour les droits des Roms (ERRC, European Roma Rights Centre) devant le Comité européen des droits sociaux en $2008^{26}$. La réclamation se fondait sur le fait que, aux yeux de l'organe requérant, les gens du voyage (Roms et autres, qu'ils soient nationaux ou étrangers) étaient victimes de discriminations dans l'accès au logement. Plusieurs articles de la Charte sociale européenne révisée étaient mis en cause : l'article 16 relatif à la protection sociale des familles (en particulier l'accès au logement, visé par le paragraphe 2), l'article 19 sur le droit des travailleurs migrants à l'assistance (là encore notamment sur le plan du logement, $\$ c)$, mais aussi la protection contre la pauvreté et l'exclusion (art. 30) et le droit au logement (art. 31). L'ensemble de ces textes était, selon l'ERRC, à lire à travers le prisme du principe de non-discrimination garanti par l'article E.

La décision sur le bien-fondé a été rendue le 26 octobre 2009, et, à la suite des observations du Gouvernement français, a fait l'objet d'une résolution du Conseil des ministres du 30 juin 2010. Le Gouvernement français, dans sa défense, a mis en avant ses efforts pour l'amélioration des conditions d'accueil des gens du voyage; il a notamment rappelé que les migrants roms en situation régulière bénéficient des structures d'accueil adaptées aux gens du voyage au même titre que les nationaux. Néanmoins, concernant les Roms en situation irrégulière qui ne peuvent s'installer sur les zones idoines, le champ reste en friche; l'organisation réclamante insiste d'ailleurs sur la persistance d' "expulsions sommaires" de ces zones dans lesquelles l'hébergement est anarchique et ne répond à aucune norme élémentaire de salubrité. Et de fait, l'accueil de ces populations roms se fait dans des conditions qui heurtent la dignité, ainsi que l'avait déjà relevé le commissaire européen aux Droits de l'homme, M. Gil Robles, dans son rapport de février $2006^{27}$, lequel avait évoqué les conditions de vie au sein des bidonvilles qui se sont constitués sur certaines périphéries parisiennes.

Deux choses sont ici à relever. En premier lieu, on peut noter que le droit au logement est certes garanti par l'article 31 de la Charte sociale européenne. Mais une lecture attentive du texte fait néanmoins apparaître l'existence non d'un droit subjectif opposable à l'État, mais bien d'une obligation positive à la charge dudit État, lequel s'engage à adopter trois séries de mesures précisées par le texte lui-même:

1) favoriser l'accès au logement d'un niveau suffisant ;

2) prévenir et réduire l'état de sans-abri en vue de son élimination progressive ;

3) rendre le coût du logement accessible aux personnes qui ne disposent pas de ressources suffisantes.

20. Voir sur ce point l'article 15 du décret 66-104 du 18 février 1966 (JO, 23 février 1966, p. 1550) et l'arrêté du 8 août 1966 (JORF, 19 août 1966, p. 7295 ).

21. Circulaire MEN 2002-101 du 25 avril 2002, publiée au Bulletin officiel de l'enseignement, $\mathrm{n}^{\circ} 10$ spécial, 25 avril $2002, \mathrm{p} .15$ sq.

22. Cette discrimination persistante des enfants roms dans l'accès à l'éducation a d'ailleurs été pointée du doigt par la Déclaration du 8 avril 2009 , intervenue dans le cadre de la Journée internationale des Roms, texte adopté par le commissaire aux Droits de l'homme du Conseil de l'Europe et différentes institutions.

23. Pour un aperçu complet de cette question de la scolarisation des enfants roms, voir notamment le site de l'Association pour l'aide à la scolarisation des enfants tsiganes: www.aset.asso.fr.

24. En effet, d'autres droits souffrent également de cette assimilation : en 2006, le Centre européen pour les droits des Roms (ERRC) avait en particulier relevé la difficulté d'accéder au marché du travail. Mais on pourrait citer également la difficulté d'obtenir un compte bancaire, un passeport, ou d'effectuer les démarches administratives les plus banales. Voire la difficulté d'obtenir une connexion Internet, liberté fondamentale aux yeux du Conseil constitutionnel français (décision 2009-580 DC du 10 juin 2009, dite HADOPI 1, $16^{\mathrm{e}}$ considérant).

25. Directive 2000/43/CE du Conseil du 29 juin 2000 relative à la mise en œuvre du principe de l'égalité de traitement entre les personnes sans distinction de race ou d'origine ethnique, JOCE L.180, 19 juillet 2000, art. 3 , $\$$ h.

26. Comité européen des droits sociaux, Réclamation 51/2008, Conseil européen des droits des Roms c. France.

27. Rapport du 16 février 2006 sur le respect effectif des Droits de l'homme en France. Il s'agissait là de l'un des derniers rapports de M. Gil Robles, qui quittait ses fonctions dans les semaines suivantes. 
La défense de l'État consiste donc à démontrer que y compris envers les populations roms, la France respecte ses obligations conventionnelles issues de la Charte, par la mise en œuvre de toute une série de politiques sociales. Néanmoins et on le sait, les obligations en la matière sont, du point de vue interne, renvoyées à la compétence du législateur. Dans une décision du 19 janvier $1995^{28}$, le Conseil constitutionnel avait établi un lien direct entre le respect de la dignité de la personne humaine et «la possibilité pour toute personne de disposer d'un logement décent ", qualifié d'objectif de valeur constitutionnelle par le Conseil. Dès lors, et ainsi que la doctrine l'a relevé, la charge de la mise en œuvre d'un tel objectif pèse sur le législateur, et non le constituant ${ }^{29}$. L'État français arguait donc de l'adoption de mesures législatives adaptées - facilitées par le fait que l'existence d'un logement décent n'était pas incompatible, aux yeux du Conseil constitutionnel, avec l'accueil sur des aires de stationnement destinées aux gens du voyage ${ }^{30}$, qu'en outre des réformes législatives avaient été introduites en droit français, en particulier la loi du 5 mars 2007 instituant le droit au logement opposable ${ }^{31}$. Lesdites réformes n'ont néanmoins que peu d'impact, le droit au logement opposable, qui permet de revendiquer effectivement la mise à disposition d'un logement, étant réservé aux nationaux et aux étrangers en situation régulière ayant déposé préalablement, et sans succès, une demande de logement social - les Roms parqués dans les bidonvilles ne répondent guère à ces conditions.

En second lieu, et c'est là le deuxième élément qui mérite d'être relevé, la situation dans ces lieux d'hébergement étant particulièrement pénible, elle permet la mise en œuvre de moyens coercitifs d'évacuation des personnes concernées. La loi du 18 mars 2003 sur la sécurité intérieure $^{32}$, développant ici une solution qui lui est antérieure, permet en effet l'expulsion en cas d' « atteinte au bon ordre, à la salubrité, à la tranquillité et à la sécurité publique ", et ce sans avoir à requérir ou à attendre d'être requis par le propriétaire du terrain (art. L. 2215-1 du Code général des collectivités locales, dit CGCT). En d'autres termes, toute atteinte à la salubrité permet à l'autorité préfectorale de décider de l'évacuation contrainte d'un site. Mieux, l'article L. 2215-1, $\$$ 4, permet même de l'ordonner sans attendre le contrôle préalable d'un juge administratif; la seule réserve est alors de pouvoir invoquer la situation d'urgence. Or, l'urgence est indéniable, compte tenu des conditions d'hygiène et de salubrité qui président dans ces camps.

En la matière, le Gouvernement français est en réalité sur un terrain glissant: arguant de ses efforts législatifs en la matière, il ne peut que composer avec la réalité, celle liée à des conditions d'existence précaires pour les
Roms, et aux évacuations forcées. Le Comité européen des droits sociaux ne pourra dès lors que reconnaître que la situation offerte aux Roms, dans la question notamment du droit au logement, n'est guère conforme aux dispositions de la Charte sociale européenne.

Reste alors la seconde approche envisageable: les Roms pris non plus en tant que gens du voyage, qu'ils soient nationaux ou étrangers, mais pris en tant qu'étrangers en tant que tels.

\section{Les Roms étrangers}

Aux termes du droit français, est étrangère toute personne qui n'a pas la nationalité française, soit qu'elle en ait une autre, soit qu'elle soit apatride (art. L. 111-1 du Code de l'entrée et du séjour des étrangers et du droit d'asile, dit CESEDA). Les Roms sont alors perçus, non plus comme gens du voyage, mais bien comme des étrangers, des personnes résidant en France à titre habituel. Leur qualité éventuelle de gens du voyage est alors subsumée par leur qualité d'étranger. Paradoxalement, et parce qu'ils ne sont pas français, il est alors possible de reconnaître l'existence d'une communauté culturelle propre - puisqu'ils ne sont pas membres du "peuple français» si cher au Constituant. Néanmoins, il serait excessif d'en déduire un régime juridique unique. Deux éléments sont ici à prendre en considération : d'une part, la nationalité des Roms (A), d'autre part, les conditions de leur départ et leur éligibilité au statut de réfugié (B).

\section{A. Un régime fonction de l'État d'origine}

Le critère de distinction fondamental est ici logique : la protection applicable à ces populations sera dépendante du point de savoir s'ils sont, ou non, ressortissants communautaires.

\section{Les Roms, ressortissants communautaires}

Les populations roms peuvent venir d'une demidouzaine d'États ayant intégré l'Union européenne, que ce soit depuis 2004 (Hongrie, Slovaquie, Slovénie, République tchèque) ou 2007 (Roumanie et Bulgarie). Quel que soit leur État d'origine, ils sont alors liés par un point commun: leur qualité de ressortissants communautaires, qui les distingue des étrangers de droit commun - et ce nonobstant la persistance de régimes transitoires pour les ressortissants des nouveaux États entrants. En conséquence, ils ne devraient subir aucune discrimination - ce que les différentes instances européennes rappellent régulièrement au demeurant ${ }^{33}$. La réalité est néanmoins

28. Décision 94-359 DC du 19 janvier 1995, Diversité de l'habitat, considérants 7 sq.

29. Voir notamment B. Jorion, «La possibilité pour toute personne de disposer d'un logement décent est un objectif de valeur constitutionnelle », Actualité juridique. Droit administratif, 1995, p. 455-462; H. Pauliat, «L'objectif constitutionnel de droit à un logement décent : vers le constat de décès du droit de propriété», Recueil Dalloz, 1995, Chronique, p. 283-287.

30. Voir notamment le $9^{e}$ considérant de la décision de janvier 1995 précitée.

31. Loi n ${ }^{\circ}$ 2007-290 du 5 mars 2007, JORF, 6 mars 2007, p. 4190.

32. Loi n ${ }^{\circ} 2003-239$ du 18 mars 2003 pour la sécurité intérieure, JORF, 19 mars 2003, p. 4761.

33. Voir notamment le Sommet européen sur les Roms, qui s'est tenu à Bruxelles le 16 septembre 2008, et qui a donné lieu à une Déclaration du même jour. 
assez différente; si les discriminations persistent partout en Europe, nous nous attacherons au cas français.

\section{a. Le principe de libre circulation}

On le sait, l'un des principes fondateurs de l'Europe communautaire est celui de la libre circulation des individus, garanti par l'article $39 \mathrm{du}$ traité fondateur. Il a été développé par un certain nombre de textes dérivés, venus mettre en ouvre le principe ; il permet aux ressortissants communautaires de bénéficier du libre franchissement des frontières intracommunautaires, mais aussi de bénéficier d'une liberté de séjour et d'installation sur le territoire des États membres. Les seules limites sont ici connues : le ressortissant communautaire est certes tenu de prouver son identité, mais à seule fin de démontrer sa qualité de ressortissant communautaire ${ }^{34}$; le séjour, qui doit être en France déclaré dans les trois mois après l'installation du ressortissant communautaire, est en outre assujetti à la possession de moyens de subsistance suffisants, qu'ils soient ou non liés à une activité professionnelle. Dans la continuité des dispositions du droit communautaire dérivé - notamment la directive 2004/ 38 du 29 avril $2004^{35}$ - et de l'article $5 \mathrm{C}$ de la Convention de Schengen, le principe en est affirmé par l'article L. 1211 CESEDA.

Or, cette obligation de ressources aboutit à une exclusion de fait des populations roms du bénéfice de la libre installation, et ce nonobstant leur qualité de ressortissants communautaires. Bien souvent, la précarité de leur situation économique ne leur permet pas de prétendre à ces moyens de subsistance, sésame du séjour sur le territoire d'un autre État membre. C'est pourquoi, persistent, aujourd'hui encore, les régulières reconduites de Roms à la frontière, en dépit de l'intégration dans l'Union européenne de plusieurs pays d'origine des populations concernées: faute de revenus, les intéressés s'exposent aux mesures d'éloignement prévues par l'article L. 511-1 CESEDA - arrêté préfectoral de reconduite à la frontière, ou obligation de quitter le territoire français lorsqu'ils ont sollicité un titre de séjour qui leur a été refusé. Les Roms se trouvent ainsi exclus, dans la pratique communautaire et française, du principe de libre circulation ${ }^{36}$.

La protection offerte à ces populations précaires est alors limitée à la protection de tout étranger, quel qu'il soit, et relève du droit commun. L'article L. 511-4 CESEDA protège, en effet, contre toute mesure d'éloignement dix catégories de personnes: l'étranger mineur, l'étranger ayant résidé en France depuis l'âge de 13 ans, l'étranger parent ou conjoint d'un ressortissant français, etc. La liste est relativement complète, mais son application aux
Roms est, là encore, assez limitée : en raison de l'endogamie qui existe au sein de la communauté rom, mais aussi du fait de séjours souvent irréguliers, le texte ne trouve guère à s'appliquer qu'aux Roms mineurs. Mais, comme souvent, on se heurte ici aux limites de la protection: dans la pratique, elle n'a vocation à protéger que les seuls mineurs isolés, les parents laissant rarement leurs enfants derrière eux lorsqu'ils font l'objet d'une mesure d'éloignement. Or, les mineurs roms isolés sont peu nombreux, leur communauté d'origine se caractérisant par une forte solidarité intra et interfamiliale.

Le constat est, dès lors, sévère: les populations roms issues d'un État communautaire ne peuvent que rarement bénéficier de l'installation en France - qu'ils soient originaires d'ailleurs d'un nouvel État membre ou d'un État historique, la situation restant ici la même pour les intéressés.

\section{b. Les Roms communautaires exerçant une activité professionnelle}

La situation pourrait être différente pour les Roms exerçant une activité professionnelle, car ici le critère des moyens de subsistance suffisants ne peut être opposé. Mais la difficulté tient alors à l'existence de régimes transitoires pour les États entrés dans l'Union européenne en 2004 et 2007 (hors Chypre et Malte). Le principe est assez simple : vis-à-vis des ressortissants de ces nouveaux États, le droit interne des «anciens» États continue de s'appliquer de préférence sur le droit communautaire.

Ce régime transitoire est limité d'un double point de vue. Rationae temporis, le maintien de mesures restreignant la libre circulation des travailleurs est limité au $1_{1}^{\mathrm{er}}$ mai 2011 pour les États entrés en 2004, et au $1^{\mathrm{er}}$ janvier 2014 pour ceux entrés en 2007. Les États peuvent, en outre, toujours décider d'accepter de façon anticipée l'application du principe de libre circulation - c'est ce qu' ont fait, par exemple, l'Espagne, le Portugal et la Finlande, dès 2006. Rationae materiae ensuite, les mesures restrictives peuvent être levées partiellement. C'est la voie choisie par la France, qui a établi dans un arrêté du 18 janvier $2008^{37}$ une liste de 150 corps de métiers se trouvant en situation de pénurie de main-d'œuvre, et pour lesquels le principe de libre circulation des travailleurs s'applique à l'ensemble des ressortissants communautaires. En conséquence, un ressortissant communautaire rom qui voudrait s'installer en France afin d'y exercer, par exemple, la profession d'électricien serait effectivement libre de le faire.

Reste, alors, la difficulté matérielle que rencontrent les populations qui nous intéressent ici : les Roms répondent

34. Voir notamment CJCE, 5 mars 1991, Giagounis, 376/89: l'arrêt, qui concernait un Grec voulant s'installer en Allemagne, rappelle que les États membres ont l'obligation de reconnaître le séjour sur leur territoire des ressortissants communautaires, la seule limite étant ici la réserve de l'ordre public, laquelle répond à une appréciation stricte.

35. Directive 2004/38/CE du Parlement européen et du Conseil du 29 avril 2004 relative au droit des citoyens de l'Union et des membres de leurs familles de circuler et de séjourner librement sur le territoire des États membres, JOCE L 204, 4 août 2007, p. 28 (art. 7, $\$ 1^{\text {er }}$, et art. 14).

36. Voir notamment J. Y. Carlier, «Les bénéficiaires et les exclus de la libre circulation des personnes ", in La libre circulation des personnes dans l'Union européenne, S. Leclerc (dir.), Bruxelles, Bruylant (Rencontres européennes, ${ }^{0}$ 12), 2009, p. 15-32.

37. Arrêté du 18 janvier 2008 relatif à la délivrance, sans opposition de la situation de l'emploi, des autorisations de travail aux ressortissants des États de l'Union européenne soumis à des dispositions transitoires, JORF, 20 janvier 2008, p. 1046 (liste des métiers figurant dans l'annexe). 
rarement aux conditions posées par le texte pour accéder à ces professions, lesquelles imposent d'avoir une formation et une qualification professionnelles qui font trop souvent défaut.

Le dispositif aboutit ainsi à une exclusion de fait de la liberté d'installation, et ce en dépit de l'entrée de la plupart des pays d'origine dans l'Union européenne. Le seul recours qui reste alors à ces populations est la mendicité sur la voie publique, ce qui se heurte à deux difficultés: d'une part, ces pratiques renforcent le sentiment d'exclusion et de rejet, d'autre part, les municipalités, depuis une dizaine d'années, tendent à adopter de plus en plus souvent ce que l'on appelle des « arrêtés antimendicité». Si le droit interne applicable aux arrêtés municipaux est relativement strict dans les conditions requises pour la légalité de ces textes, reste que nombre de grandes villes ont mis en place une police administrative restrictive, qui frappe les personnes en situation de fragilité économique, et notamment les Roms. Ces derniers se trouvent en situation d'être pénalement réprimés, ou d'être relégués dans les périphéries, ce qui accentue leur précarité.

\section{Les Roms venant d'un État tiers}

La problématique se pose en des termes différents s'agissant des populations roms issues d'États non membres de l'Union européenne, dans la mesure où s'applique alors le droit commun des étrangers. Les conditions d'entrée sont celles fixées par l'article L. 211-1 CESEDA: passeports et visas lorsqu'ils sont exigés, justificatifs prouvant la possession de moyens de subsistance, éventuels documents idoines pour exercer une activité professionnelle, etc. Jusque très récemment, un visa restait obligatoire pour les ressortissants de la Serbie, de l'ancienne République yougoslave de Macédoine et du Monténégro; cette obligation a été levée par un règlement du Conseil de l'Union européenne du 30 novembre 2009, entré en vigueur le 19 décembre $2009^{38}$. Aujourd'hui, l'obligation persiste néanmoins pour les ressortissants de l'Albanie et de la Bosnie-Herzégovine, ainsi que pour les résidents du Kosovo.

Dans toutes les hypothèses, la venue et l'installation en France restent soumises à l'obligation, que l'on retrouve pour les ressortissants communautaires, de disposer des fonds suffisants pour subsister sans avoir à attendre de recours de la part des autorités publiques françaises. S'agissant de populations souvent pauvres, le contrôle de ces moyens, qui repose soit sur le consulat dans le pays d'origine, soit sur les autorités internes à la frontière ou sur le territoire, soulève les problèmes déjà évoqués précédemment. Et, de fait, le Rapport 2008 de la Commission nationale consultative des Droits de l'homme (CNCDH) met en exergue le fait que les autorités françaises se fondent sur ce motif pour refouler les Roms. Les mêmes causes produisent les mêmes effets.

\section{B. La difficile éligibilité des Roms au statut de réfugié}

Si le droit commun des étrangers paraît donc peu adapté à la situation des populations roms, reste peut-être la voie du régime dérogatoire, par le biais de la notion de réfugié. Et de fait, le rapport de l'Office français de protection des réfugiés et apatrides (OFPRA) mentionne, chaque année, quelques centaines de demandes présentées par des Roms, venus du Kosovo principalement.

En France, l'obtention d'un statut juridique protecteur pour les personnes pouvant craindre avec raison des persécutions trouve trois fondements distincts.

En premier lieu, intervient le statut international, issu de la Convention de Genève du 28 juillet 1951 portant statut des réfugiés. La jurisprudence française était traditionnellement restrictive dans son acception de la définition du réfugié, le limitant aux personnes pouvant se prévaloir de persécutions perpétrées, ou à tout le moins tolérées volontairement, par les autorités publiques ${ }^{39}$; cette restriction a néanmoins aujourd'hui été rejetée par une loi de 2003. Reste donc la définition internationale du statut en particulier les cinq chefs limitatifs de persécution que sont la race, la nationalité, la religion, les opinions politiques ou l'appartenance à un certain groupe social. Les Roms, s'ils peuvent prouver répondre à l'un de ces motifs (notamment la nationalité) et craindre avec raison d'être persécutés à titre individuel, peuvent espérer une reconnaissance du statut de réfugié, qui ouvrira la voie d'un titre de séjour de dix ans (art. L. 314-11, $8^{\circ}$ CESEDA).

En second lieu, le statut de réfugié peut être reconnu sur le fondement de l'existence d'un combat pour la liberté, au sens de la Constitution française ${ }^{40}$. La condition est néanmoins ici restrictive; si l'asile au sens de la Constitution trouve rarement à s'appliquer, il n'a jamais été reconnu pour des populations roms, qui sont victimes bien plus souvent de préjugés, et non des conséquences d'un putatif combat pour la liberté.

Enfin et en troisième lieu, le droit français a intégré la Protection subsidiaire fondée sur une directive

38. Règlement (CE) 2009/1244 du Conseil modifiant le Règlement (CE) 2001/539 fixant la liste des pays tiers dont les ressortissants sont soumis à l'obligation de visa pour franchir les frontières extérieures des États membres et la liste de ceux dont les ressortissants sont exemptés de cette obligation, JOUE L.336, 18 décembre 2009. L'exemption de visa ne concerne néanmoins que les titulaires d'un passeport biométrique.

39. Une telle approche était due à une lecture combinée des articles $1 \mathrm{~A} 2$ et $1 \mathrm{C} 1$ de la Convention de Genève, selon lesquels, d'une part, le statut n'est possible que dans la mesure où l'individu ne peut obtenir de protection de son État de nationalité (art. 1A2) et, d'autre part, le fait de retrouver volontairement cette protection induit la fin du statut international (art. 1C1). La France avait donc imposé que les persécutions ne pouvaient émaner que des autorités publiques; elle avait assoupli son approche en considérant que lesdites persécutions pouvaient émaner de personnes privées dans la mesure où les agissements étaient volontairement tolérés par les pouvoirs publics (revirement dû à un arrêt du Conseil d’État: CE, 27 mars 1983, Dankha, Lebon, 1983, p. 220). C'est cette acception qui a été repoussée par l'évolution législative de 2003.

40. Préambule de la Constitution du 27 octobre 1946, alinéa 4: « tout homme persécuté pour son action en faveur de la liberté a droit d'asile sur les territoires de la République». Le texte est intégré dans le bloc de constitutionnalité, c'est-à-dire qu'il a pleine valeur constitutionnelle en France (ce qu'a confirmé notamment le Conseil constitutionnel dans sa décision 93-353 DC du 13 août 1993, Loi relative à l'immigration, 84 ${ }^{\mathrm{e}}$ considérant). 
communautaire de $2004^{41}$. Envisagée dans le cas où les deux premiers fondements n'ont pu permettre de protection, la protection subsidiaire est soumise à trois conditions alternatives : le fait d'être exposé à la peine de mort, le fait d'être exposé à des traitements contraires à l'article 3 de la Convention européenne des Droits de l'homme (torture, peines et traitements inhumains ou dégradants), ou le fait d'être un civil pris dans une situation de conflit armé. Or là encore, le texte trouve difficilement à s'appliquer pour des Roms. La peine de mort est prohibée par les Protocoles VI et XIII de la Convention EDH, textes assez largement ratifiés en Europe. Certes, il ne faudrait pas en déduire une exclusion d'office des Roms, néanmoins, les hypothèses restent peu probables. Concernant des situations de conflits armés, de la même façon, l'applicabilité aux Roms se rapproche de la pure théorie, les intéressés étant certes victimes de discriminations, mais qui ne sont pas liées à des conflits armés. Les évolutions jurisprudentielles de la Cour de justice de l'Union européenne n'ont donc ici guère d'intérêt ${ }^{42}$. Reste la troisième hypothèse d'octroi de la protection subsidiaire, celle liée à des traitements prohibés par l'article 3 de la Convention EDH. C'est sans doute ici que les Roms ont le plus vocation à trouver un soutien; néanmoins, l'application reste difficile. En particulier, la protection subsidiaire impose que les traitements dépassent un seuil de gravité minimal qui n'existe pas toujours, s'agissant de brimades et de discriminations. Ainsi, l'analyse des décisions récentes rendues par la Cour nationale du droit d'asile (CNDA), juridiction compétente en la matière, montre que, si les comportements inacceptables existent et sont reconnus comme tels, ils n'aboutissent pas pour autant à la reconnaissance d'un statut ${ }^{43}$.

Car en réalité, l'obstacle à surmonter, dans le cas des demandes présentées par des Roms, qu'ils soient de Serbie ou d'ailleurs, reste l'imbrication des motifs de leur départ: en effet, ces populations sont victimes à la fois d'une situation économique précaire, de discriminations fondées sur leur origine ${ }^{44}$, parfois de brimades ou de violences, mais qui n'atteignent alors pas toujours le seuil minimal de gravité exigé par les autorités françaises pour leur reconnaître un statut dérogatoire au droit commun. S’y ajoute la difficulté liée à leur État de provenance, considéré comme sûr. Dès lors, la seule solution passe par une évolution de la jurisprudence interne française.

\section{Les Roms et la notion de pays d'origine sûr}

Depuis le Sommet européen de Londres et ses conclusions adoptées par les ministres le $1^{\text {er }}$ décembre $1992^{45}$, l'Europe a fait place à une notion nouvelle, celle de pays d'origine sûr. L'idée est qu'un étranger venant d'un tel État ne craint pas en principe de persécution et n'a donc pas à bénéficier des dispositions protectrices relatives aux réfugiés et/ou bénéficiaires de la protection subsidiaire. En droit interne, cela aboutit à ce que les ressortissants de tels États voient leur demande d'asile examinée selon une procédure accélérée, et peuvent se heurter à un refus d'entrée sur le territoire national (art. L. 741-4 CESEDA). Cette liste, remodelée le 4 décembre 2009, comprend notamment l'Arménie, la Bosnie-Herzégovine, la Croatie, l'ancienne République yougoslave de Macédoine et la Serbie. Matériellement, cela induit que les Roms originaires de ces États - comme d'ailleurs les Roms originaires des États membres de l'Union européenne - voient leurs chances d'obtenir une protection de l'OFPRA réduites, à défaut d'être inexistantes. Il est certes possible de se voir reconnaître le statut de réfugié en dépit d'un État d'origine sûr (et l'on pense ici notamment aux ressortissants turcs, la Turquie ayant été inscrite sur la liste des pays d'origine sûr en 2009); néanmoins, l'admission en France peut être refusée, et la demande d'asile aura peu de chances de succès puisque le pays d'origine est réputé ne tolérer aucune persécution.

En l'état, cela veut dire qu'en France tout au moins, hors le cas de l'Albanie ${ }^{46}$, les Roms pourront difficilement obtenir la reconnaissance d'une protection idoine, d'une part en raison de leur État d'origine, d'autre part en raison des faits mêmes qui fondent leurs demandes. Pour autant, il ne faudrait pas en déduire une exclusion systématique des Roms de toute forme de protection. Ainsi en juillet 2000, une quarantaine de Roms venus de Hongrie étaient arrivés à Strasbourg et, après avoir manifesté devant les locaux du Conseil de l'Europe, avaient déposé une demande d'asile auprès des autorités préfectorales. La Hongrie n'était alors pas encore membre de l'Europe communautaire, mais elle était déjà considérée comme

41. Directive 2004/83/CE du 29 avril 2004, transposée dans le CESEDA français dès son adoption, et dont les dispositions figurent aujourd'hui aux articles L. 712-1 sq. dudit Code.

42. La Cour a, en effet, précisé que dans un tel cas le critère d'individualisation des risques pouvait céder face à la généralisation de la violence : voir CJCE, 17 février 2009, Époux Elgafaji, aff. 467/07. Néanmoins, les Roms ne sont pas à proprement parler confrontés à une situation de conflit armé aujourd'hui en Europe.

43. Voir, pour exemples: CNDA, 12 février 2009, $M^{\text {lle }} D^{\star}, 634850$, pour une ressortissante de Serbie d'origine rom (insultes et coups de la part de policiers); CNDA, 18 décembre 2008, Directeur général de l'OFPRA c. $M^{\text {Ile }} B^{*}, 595669$, pour une femme ressortissante de Macédoine.

44. Or, il a été relevé très tôt que l'imbrication des motifs économiques et de ceux relevant du droit des réfugiés peut aboutir à exclure de la protection: Cour EDH, 20 mars 1991, Cruz Varas c. Suède, A. 201; voir la note de G. Cohen-Jonathan, "De l'effet juridique des mesures provisoires dans certaines circonstances et de l'efficacité du droit de recours individuel : à propos de l'arrêt Cruz Varas ", Revue universelle des Droits de l'homme, 1991, p. 205-221; le commentaire d’E. Garcia de Enterria, «De la légitimité des mesures provisoires prises par la Commission et la Cour européennes des Droits de l'homme : l'affaire Cruz Varas", Revue trimestrielle des Droits de l'homme, n' 11, 1992, p. 251-280.

45. On trouve ce texte dans: E. Guild et J. Niessen, The Developing Immigration and Asylum Policies of the European Union. Adopted Conventions, Resolutions, Recommendations, Decisions and Conclusions, La Haye - Londres - Boston, Kluwer Law International, 1996, p. 141,161 et 177.

46. L'Albanie avait primitivement été inscrite sur la liste des pays d'origine sûr par l'OFPRA. Le Conseil d'État, saisi du contrôle de la liste, l'en avait néanmoins retirée, estimant que compte tenu de l'instabilité politique régnant dans le pays, l'Albanie ne présentait pas à ce jour les caractéristiques de démocratie, d'État de droit et de respect des libertés fondamentales qu'impose la qualité de pays d'origine sûr (CE, 19 février 2008, Association Forum Réfugiés, 295443, mentionné dans les tables du Recueil Lebon, Lebon 2008. T., p. 774). 
un pays d'origine sûr; pourtant, les autorités françaises avaient effectivement reconnu le statut de réfugié, admettant l'existence de persécutions fondées sur la race $^{47}$. Toutefois, un exemple ne saurait faire une règle, et la réalité reste celle d'une accumulation d'obstacles opposés aux Roms avant même l'examen de toute demande au fond. Et ces obstacles ne disparaissent pas lorsque le fond est effectivement appréhendé.

\section{Quelle(s) persécution(s) pour les Roms?}

Les critères ordinaires du droit français des réfugiés trouvent difficilement à s'appliquer pour des populations roms, ce qu'atteste le faible nombre d'étrangers obtenant une réponse positive de la part de l'OFPRA. La difficulté tient ici au seuil de gravité, mais aussi à une condition constante, commune tout à la fois au statut de réfugié et à la protection subsidiaire : celle de l'individualisation des craintes de persécution ${ }^{48}$. Le demandeur doit prouver qu'il est personnellement l'objet des faits qu'il invoque; or, le problème des Roms est que les agissements en cause ne sont pas toujours ciblés sur un individu et concernent bien souvent l'ensemble des Roms. Dès lors, faute de répondre à la condition d'individualisation, la demande risque d'être rejetée. Le droit des réfugiés, au sens strict comme au sens large, a vocation depuis la seconde guerre mondiale à protéger des individus et non des groupes d'individus, voire des populations entières ${ }^{49}$.

La solution serait d'introduire une évolution, en faveur des groupes qui font l'objet de persécutions. L'évolution pourrait venir, ici, de la Cour EDH et de sa jurisprudence relative à l'application de l'article $3 \mathrm{CEDH}$ aux mesures d'éloignement des étrangers. La Cour EDH considère, depuis de longues années, qu'une mesure d'éloignement ne doit pas aboutir à exposer l'étranger, dans le pays de renvoi, à des traitements prohibés ${ }^{50}$. Or, en 2007, est apparue dans la jurisprudence européenne une évolution intéressante. Dans un arrêt Salah Sheek ${ }^{51}$, la Cour a en effet admis de retenir la protection de l'article 3 $\mathrm{CEDH}$ pour un requérant somalien non qui invoquait des persécutions personnelles, mais qui expliquait être membre d'une minorité (la tribu des Ashrafs) qui, elle, faisait l'objet d'une persécution systématique. Le requérant avait démontré qu'il se trouvait dans la même situation que l'ensemble des garçons de son village, dont il ne se distinguait en rien; tous subissaient le même traitement, en raison de leur origine tribale. Le principe d'individualisation des craintes aurait voulu que la Cour rejette sa demande; or la Cour, bien au contraire, a retenu l'argument, relevant l'appartenance du requérant à un groupe minoritaire particulièrement menacé. Et la Cour de noter que les intéressés sont « des proies faciles, comme l'ensemble des familles ashrafs vivant dans le même village» (\$148); elle considère qu'exiger une preuve de risques personnels aboutirait, en l'espèce, à rendre la protection de la Convention illusoire ${ }^{52}$.

Cette jurisprudence a été reprise et systématisée l'année suivante par la Grande Chambre, dans l'arrêt Saadi c. Italie de $2008^{53}$. Saisie de la requête d'un ressortissant tunisien qui se trouvait en Italie et était en voie d'être expulsé vers son pays d'origine à raison d'accusations de terrorisme, la Cour répond par un dictum clair : «Dans les affaires où un requérant allègue faire partie d'un groupe systématiquement exposé à une pratique de mauvais traitements, la Cour considère que la protection de l'article 3 de la Convention entre en jeu lorsque l'intéressé démontre $[. .$.$] qu'il y a des motifs sérieux et avérés$ de croire à l'existence de la pratique en question et à son appartenance au groupe visé (\$132).»

Et la Cour d'en déduire que, lorsque ces traitements prohibés sont attestés par des rapports émanant notamment d'organisations non gouvernementales (ONG), une violation de l'article 3 du fait de l'éloignement de l'étranger requérant peut être retenue.

47. Les faits sont rapportés par l'étude de la Commission nationale consultative des Droits de l'homme (CNCDH), La situation des Roms et des gens du voyage, 7 février 2008, p. 21 (disponible sur le site www.cncdh.fr).

48. Nous mettrons ici de côté la question de l'individualisation des craintes dans le cadre de la mise en œuvre de la protection subsidiaire fondée sur la situation d'un civil pris dans une situation de conflit armé. Si la jurisprudence précédemment évoquée de la CJCE permet effectivement de repousser le critère individuel en cas de violence généralisée, nous avons vu que l'hypothèse même trouvait difficilement à s'appliquer aux populations roms, qui aujourd'hui ne se trouvent que rarement aux prises avec un conflit armé.

49. Les premiers instruments internationaux visaient en effet des populations entières: Arméniens fuyant le génocide de 1916, Russes fuyant la révolution de 1917, etc. Après 1945 néanmoins, les instruments ne se sont plus limités à des nationalités particulières et ont opté pour une approche universaliste ; le contrepoids a alors consisté à imposer la preuve de craintes personnelles, à la charge de tout demandeur.

50. Il s'agit de l'application dite par ricochet de l'article 3 CEDH, largement développée depuis l'arrêt fondamental Cour EDH, 7 juillet 1989 , Soering c. Royaume Uni, 14038/88, $\$ 82$. Pour une approche complète de cette jurisprudence et de ses incidences, voir J. Julien-Laferrière, «L'application par ricochet de l'article $3 \mathrm{CEDH}$ : l'exemple des mesures d'éloignement des étrangers ", in La portée de l'article 3 de la Convention européenne des Droits de l'homme, C.-A. Chassin (dir.), Bruxelles, Bruylant, 2006, p. 141-157.

51. Cour EDH, 11 janvier 2007, Salah Sheek c. Pays-Bas, 1948/04 (en anglais).

52. $\$ 148:$ : The Court would add that, in its opinion, the applicant cannot be required to establish the existence of further special distinguishing features concerning him personally in order to show that he was, and continues to be, personally at risk. In this context it is true that a mere possibility of ill-treatment is insufficient to give rise to a breach of Article 3. Such a situation arose in the case of Vilvarajah and Others v. the United Kingdom, where the Court found that the possibility of detention and ill-treatment existed in respect of young male Tamils returning to Sri Lanka. The Court then insisted that the applicants show that special distinguishing features existed in their cases that could or ought to have enabled the United Kingdom authorities to foresee that they would be treated in a manner incompatible with Article 3 (judgment cited above, p. 37, \$111-112). However, in the present case, the Court considers, on the basis of the applicant's account and the information about the situation in the "relatively unsafe" areas of Somalia in so far as members of the Ashraf minority are concerned, that it is foreseeable that on his return the applicant would be exposed to treatment in breach of Article 3. It might render the protection offered by that provision illusory if, in addition to the fact of his belonging to the Ashraf - which the Government have not disputed -, the applicant were required to show the existence of further special distinguishing features."

53. Cour EDH, Gde ch., 28 février 2008, Saadic. Italie, 37201/06. 
Cette acception est riche de potentialités, s'agissant de populations victimes, de façon systématique, de traitements contraires à l'article $3 \mathrm{CEDH}$. Pour les Roms, cela pourrait leur permettre de faire valoir, auprès des autorités françaises, la mise en œuvre de la protection subsidiaire, sans que puisse leur être opposée l'absence éventuellement d'individualisation des craintes invoquées. L'absence de personnalité pourrait alors être compensée par la présentation de rapports d'ONG - voire d'organisations internationales comme l'Union européenne ou le Conseil de l'Europe - sur la situation des Roms dans l'État d'origine. L'individu serait alors subsumé par son groupe d'appartenance.
Néanmoins, une telle évolution de la jurisprudence interne française reste à ce jour une simple théorie, à laquelle ni l'OFPRA ni le juge administratif n'ont donné de concrétisation jurisprudentielle. On peut le regretter, tant du point de vue de la logique juridique (l'harmonisation de la position des juges français avec celle de la Cour $\mathrm{EDH}$ ) que sur le plan humain (le rejet de cette extension prive les Roms d'espérer raisonnablement obtenir une protection de l'OFPRA); il appartient ici tant aux avocats, aux associations d'aide qu'aux universitaires, de montrer aux organes compétents le chemin déjà emprunté par la Cour européenne - en se fondant, par exemple, sur les récents travaux sur la question menés par l'Agence européenne des droits fondamentaux ${ }^{54}$. 
